\title{
Multi-Beam Scanning Electron Microscope Design
}

\author{
Pieter Kruit ${ }^{1}$ and Yan Ren ${ }^{1}$
}

1. Delft University of Technology, Department of Imaging Physics, Delft, The Netherlands.

Electron beam instruments in which not one, but many electron beams travel in parallel were first conceived for applications in the semiconductor industry. For electron beam lithography to play a role in the production of integrated circuits, multi-beam technology is essential for obtaining sufficient throughput. At present day feature sizes electron beam inspection of full wafers will only be possible with multi-beam technology. These kind of instruments are under development in several places in the world.

About 10 years ago we started to look at the possibilities to create a multi-beam scanning electron microscope [1] because we were convinced that a multi beam system with a higher throughput can have a big impact in microscopy as well, for diverse applications. This idea was confirmed with the rise of 3D imaging and the obvious need for higher imaging rates in that field [2]. A rough estimate is enough to show the potential impact: To form a 3D image of a $400 \mu \mathrm{m} * 400 \mu \mathrm{m} * 1000 \mu \mathrm{m}$ brain volume, a single beam SEM, needs something like 400 days, depending on the choice of imaging conditions. A 200 beam MBSEM should reduce this to 2 days if it is able to image without reduction of beam quality or detection efficiency. Regular 2D imaging could also benefit from fast imaging, especially in samples where the information of interest is scarce. Large area imaging in combination with large computer memory and modern image recognition techniques would open up a new way of doing microscopy, which I refer to as "Google map microscopy".

In a few successive $\mathrm{PhD}$ projects we transformed a FEI Novanano column into an instrument with an array of $14 \times 14$ focused electron beams on the sample. To keep it simple, only the original source module was replaced by one in which the beam from the Schottky source is divided into 196 separate sub-beams. Each beam has about $1 \mathrm{nA}$ of current and the same brightness as the original single beam $[3,4]$. The array of source images is subsequently demagnified by the existing SEM optics. The pitch of the primary beams at the sample is in the range of $0.3 \sim 4 \mu \mathrm{m}$. From an optics point of view, the first challenge is to design and build a lens array where each lens forms a stigmatic and correctly focused image of the source without the need for individual focusing or stigmation. The second challenge is to get the array demagnified onto the sample with introduction of neglegable off-axis aberrations from the imaging lenses. The final challenge is to get the signal from each individual beam on the sample to an individual detector.

Secondary electrons can be accelerated back into the objective lens by biasing the sample with a negative potential. The optics of the SEM can then be used as in a low energy electron microscope or photo-emission electron microscope to image the secondary electron "image", consisting of 196 points, onto a detector plane. Here the signals can be collected by an array of photo multipliers or a fast camera. We are also experimenting with the transmission signal which is collected by putting the thin sample directly on a luminescent YAG screen. The separated light signals are imaged onto a camera with a high NA objective lens developed for in-situ correlative microscopy [5], see figure 1. This give surprisingly high contrast images, best compared to brightfield TEM images at low accelerating voltage, see figure 2. 
Recently Zeiss developed a commercially available multi-beam scanning electron microscope [6] also based on splitting the beam in the source and demagnifying the array of source images with regular magnetic macro-lenses. The imaging mode is secondary electron imaging. This will allow the early customers to develop ways of working with the high data rates, find first applications and detect the benefits and shortcomings of this first generation MBSEM. There are still many design parameters to optimize such as number of beams and pitch between the beams, there are first generation problems to minimize such as off-axis aberrations, detection efficiency loss and crosstalk and there are challenges to face such as the development of a backscatter detection mode. [7]

\section{References:}

[1] Y. Zhang and P. Kruit; J. Vac. Sci. Technol. B 25 (2007), 2239

[2] K,L.Briggman and D.D.Bock; Current opinion in neurobiology 22 (2012), 154-161

[3] A. Mohammadi-Gheidari; P.Kruit, Nucl. Instrum. and Meth. in Phys. Res. A 645 (2011), 60

[4] A. Mohammadi-Gheidari; C. W. Hagen and P. Kruit, J. Vac. Sci. Technol. B28 (2010), 1071

[5] A.C. Zonnevylle et al; J Microsc. 251 (2013), 58-70

[6] A.L.Eberle et al; J Microsc. 259 (2015), 114

[7] The rat pancreas tissue of figure 2 was supplied by UMC Groningen (Giepmans group).
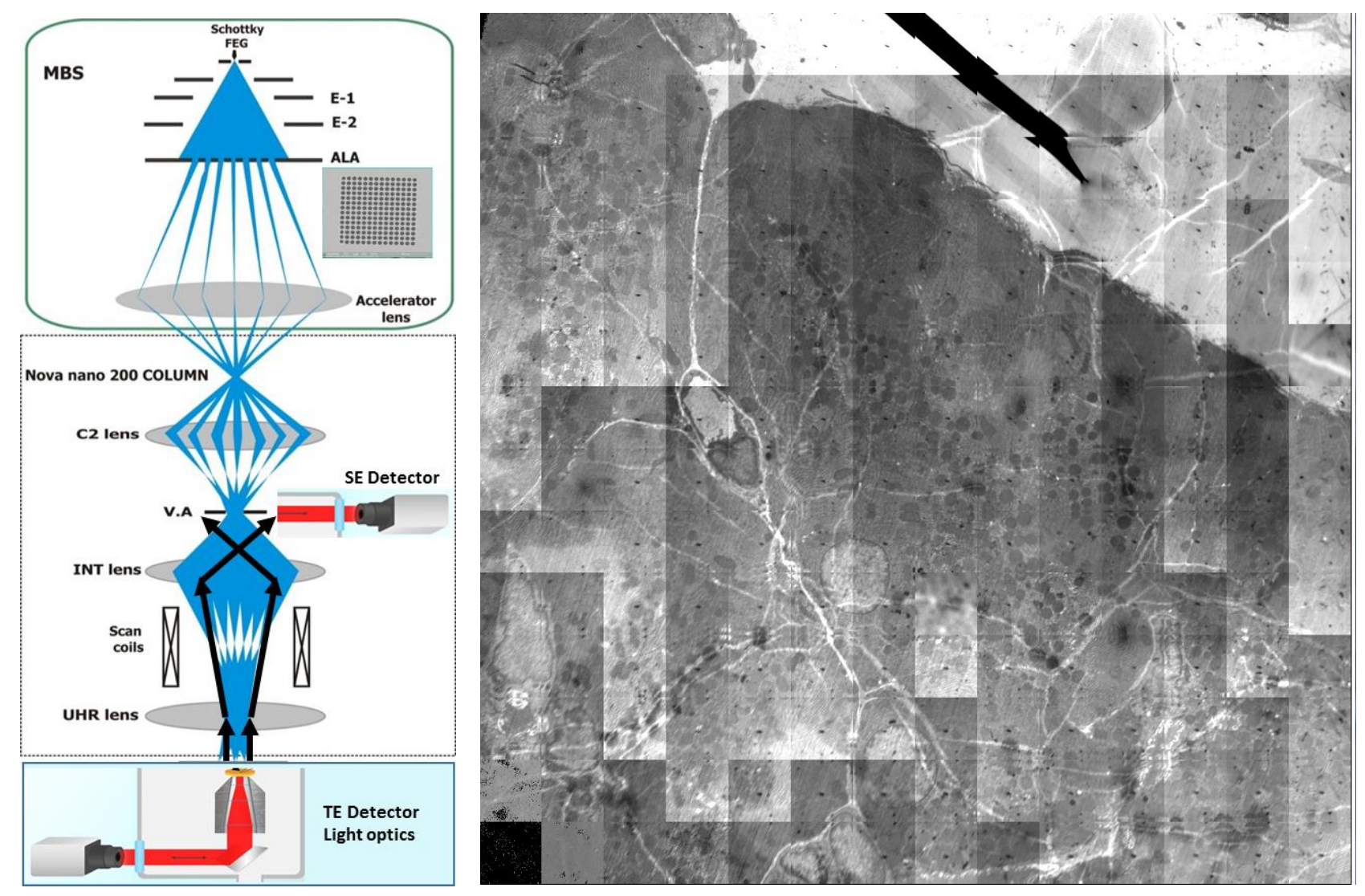

Figure 1. Schematic design of the Delft Multi-Beam SEM.

Figure 2. 196 images of rat pancreas tissue, obtained in transmission mode. To show the multi-beam principle, the images have not yet been corrected for intensity differences and stitching errors. Total size of this image is $50 \mu \mathrm{m}$. 